\title{
A SUPPLEMENT TO “SZEGÖ KERNEL FUNCTION ON SOME DOMAINS OF INFINITE CONNECTIVITY”,
}

\author{
By Mitsuru Ozawa
}

1. Let $D$ and $\mathfrak{B}(D)$ be an $n$-ply connected analytic domain and the class of regular functions in $D$ whose moduli are bounded by the value 1 , respectively. In $\mathfrak{B}(D)$ there exists, up to a rotation, a unique extremal function by which the maximum $\max \mathfrak{B}(D)\left|f^{\prime}\left(z_{0}\right)\right|$ for a fixed point $z_{0}$ is attained. This extremal function $F\left(z, z_{0}\right)$ maps $D$ onto the $n$ times covered unit disc [1], [3], [4], [9], [11]. In $\mathfrak{B}(D)$ there exists an infinite number of essentially different functions which map $D$ onto the $n$ times covered unit disc [2], [5], [8]. Evidently the valence function $n(w)$ of these functions is equal to zero for any $w$ lying in the exterior of the unit disc and $n$ for any $w$ lying in the interior of the unit disc. It is an important problem to define the meaning that the image domain of a mapping function covers a domain infinitely many times. Various attempts was made hitherto of this tendency. Among them Heins' notions of "of type $B l_{1}$ " and "of type $B l$ " give two important answers [6].

In the present paper we shall prove that the extremal function $F\left(z, z_{0}\right)$ is of type $B l_{1}$ with at most one exceptional point at which the map is of type $B l$ in a case of an infinitely connected domain defined in later part. Is there a maximal planar domain for the class $\mathfrak{B}(D)$ on which the class of any extremals belonging to the class of type $B l$ is not empty while no extremal function of type $B l_{1}$ exists? This problem is not yet settled, but it is very plausible to conjecture that there is such a domain. As far as we are aware, no systematic study has been made of this tendency for the class $\mathfrak{B}(D)$ in the case of infinitely connected domains.

2. Let $D$ be a domain of finite type and of infinite island type defined in [10]. Then we have

$$
\sum_{\nu=1}^{\infty} \frac{r_{\nu}}{a_{\nu}^{2}-r_{\nu}^{2}}<\infty
$$

which is equivalent to a fact that the function $1 / z$ is of class $L^{2}$. To proceed further we assume that the function $1 / z^{2}$ belongs to the class $L^{2}$, which is equivalent to the condition (C) defined in [10]. Thus it is allowable to make free use of our earlier results.

Next we make some preparations on the positive or bounded harmonic functions in $D$. It is evident that there is no bounded harmonic function in $D$ with vanishing boundary value on each $C_{n}$. Further, there is in $D$ only one

Received April 20, 1961. 
singular minimal positive harmonic function with vanishing boundary value on each $C_{n}$. For the definitions of these notions see [6]. This is not evident, since, in general, there is a domain for which this does not hold. In the first place we can map $D$ onto a domain with an infinite number of slits lying on the real positive axis by the Riemann mapping theorem. Then we can apply the reasoning due to Kjellberg [7] sec. 23, since all situations needed are invariant by an arbitrary conformal mapping. For this minimal positive harmonic function $m(z)$ we have the following fact:

$$
\lim _{z \rightarrow 0} m(z)=+\infty
$$

along a path lying in an angular domain $\Theta: \pi / 2+\varepsilon<\arg z<3 \pi / 2-\varepsilon$ defined in $[10]$.

Let $F\left(z, z_{0}\right)$ be the extremal function in the class $\mathfrak{B}(D)$. Then we have the representation

$$
F\left(z, z_{0}\right)=\frac{k\left(z, z_{0}\right)}{l\left(z, z_{0}\right)}
$$

and the uniqueness of the extremal function up to the rotation. And further we have the analyticity of $F\left(z, z_{0}\right)$ and $\left|F\left(z, z_{0}\right)\right|=1$ on each $C_{n}$ and the limiting behaviors

$$
k\left(z, z_{0}\right)-\overline{k_{1}\left(z_{0}\right)}=O(|z|) \text { and } l\left(z, z_{0}\right)+l_{1}\left(z_{0}\right)=O(|z|)
$$

in the angular domain $\Theta$. Thus we have

$$
F\left(z, z_{0}\right)+\frac{\overline{k_{1}\left(z_{0}\right)}}{l_{1}\left(z_{0}\right)}=O(|z|)
$$

there. The function $k_{1}\left(z_{0}\right) / l_{1}\left(z_{0}\right)$, as a function of $z_{0}$, is also bounded:

$$
\left|\frac{k_{1}\left(z_{0}\right)}{l_{1}\left(z_{0}\right)}\right| \leqq 1
$$

and is regular in $D$. If its modulus is equal to 1 at an inner point $z_{0}$, then it is a constant of modulus 1 . However it was also proved already that $k_{1}\left(z_{0}\right)$ tends to a positive constant $\left\|k_{1}\left(z_{0}\right)\right\|^{2}$, when $z_{0}$ tends to the origin in $\Theta$ and $l_{1}\left(z_{0}\right)$ tends to the infinity simultaneously. Therefore we arrive at a contradiction. Thus we have a fact that

$$
0 \leqq\left|\frac{k_{1}\left(z_{0}\right)}{l_{1}\left(z_{0}\right)}\right|<1
$$

at any point $z_{0}$ in $D$.

Let $a$ be any constant lying in the unit disc $\{|w|<1\}$. Then we have

$$
-\log \left|\frac{F\left(z, z_{0}\right)-a}{\varepsilon_{2} 1-\bar{a} F\left(z, z_{0}\right)}\right|=\sum_{j=0}^{\infty} g\left(z, z_{j}\left(z_{0}\right)\right)+\mu\left(z_{0}\right) m(z),
$$

where the summation is taken over all the $a$-points $z_{j}\left(z_{0}\right)$ of $F\left(z, z_{0}\right)$ and $g(z, p)$ is the Green function of $D$ with its singularity $p$ and $\mu\left(z_{0}\right)$ is a non-negative constant. If $a$ is not equal to $-\overline{k_{1}\left(z_{0}\right)} / l_{1}\left(z_{0}\right)$, then $\mu\left(z_{0}\right)$ must be equal to zero. 
Indeed, suppose that $\mu\left(z_{0}\right)$ is not equal to zero, then the right hand side term tends to $+\infty$ but the left hand side term is bounded, when $z$ tends to the origin in $\Theta$. This is absurd. This leads to a relation

$$
-\log \left|\frac{F\left(z, z_{0}\right)-a}{1-\bar{a} F\left(z, z_{0}\right)}\right|=\sum_{j=0}^{\infty} g\left(z, z_{j}\left(z_{0}\right)\right)
$$

and

$$
\lim _{z \rightarrow 0} \sum_{j=0}^{\infty} g\left(z, z_{j}\left(z_{0}\right)\right)<\infty
$$

in $\Theta$. If $a$ is equal to $-\overline{k_{1}\left(z_{0}\right)} / l_{1}\left(z_{0}\right)$, then we cannot yet say that $\mu\left(z_{0}\right)$ reduces to zero. However it must satisfy a system of simultaneous equations

$$
\sum_{\jmath=0}^{\infty} \omega_{\nu}\left(z_{j}\left(z_{0}\right)\right)+\mu\left(z_{0}\right) \frac{1}{2 n} \int_{C_{\nu}} \frac{\partial}{\partial n} m(z) d s \equiv 0(\bmod 1), \nu=1,2, \cdots,
$$

where $\omega_{\nu}(z)$ is the harmonic measure $\omega\left(z, C_{\nu}, D\right)$.

Theorem. Any extremal function $F\left(z, z_{0}\right)$ is of type $B l_{1}$ for the unit disc $|w|<1$ with at most one exceptional point $w=-\overline{k_{1}\left(z_{0}\right)} / l_{1}\left(z_{0}\right)$, at which the map is locally of type $B l$.

3. It should be here remarked that the system of equations reduces to a simpler one in our case. We shall state only our result:

The image curve lying on the unit circle $|w|=1$ of each boundary circle $C_{\nu}$ by the function $F\left(z, z_{0}\right)$ circumscribes just once the origin, and further the system of equations

$$
\sum_{j=0}^{\infty} \omega_{\nu}\left(z_{j}\left(z_{0}\right)\right)+\mu\left(z_{0}\right) \frac{1}{2 \pi} \int_{C_{\nu}} \frac{\partial}{\partial n} m(z) d s=1, \quad \nu=1,2, \cdots
$$

for $a=-\overline{k_{1}\left(z_{0}\right)} / l_{1}\left(z_{0}\right)$ is valid. These equations have a system of solutions $z_{j}\left(z_{0}\right)$ for any given $z_{0}$ and $-\overline{k_{1}\left(z_{0}\right)} / l_{1}\left(z_{0}\right)$.

\section{REFERENCES}

[1] Ahlfors, L. V., Bounded analytic functions. Duke Math. Journ. 14 (1947), $1-11$.

[2] Bieberbach, L., Über einen Riemannschen Satz aus der Lehre von der konformen Abbildung. Sitz. Berliner Math. Gesell. 24 (1925), 6-9.

[3] Garabedian, P. R., Schwarz's lemma and the Szegö kernel function. Trans. Amer. Math. Soc. 67 (1949), 439-459.

[4] Garabedian, P. R., AND M. Schiffer, On existence theorems of potential theory and conformal mapping. Ann. of Math. 52 (1950), 164-187.

[5] GRUNSKY, H., Über die konforme Abbildung mehrfach zusammenhängender Bereiche auf mehrblättrige Kreise. Sitz. Preuss. Akad. Wiss. (1937), 1-9.

[6] Herns, M., On the Lindelöf principle. Ann. of Math. 55 (1955). 296-317.

[7] KJELLbERG, B., On certain integral and harmonic functions. A study in minimum modulus. Thèse Uppsala (1948) $64 \mathrm{pp}$. 
[8] MoRI, A., Conformal representation of multiply connected domain on many sheeted disc. Journ. Math. Soc. Japan 2 (1951), 198-209.

[9] NeHArI, Z., On bounded analytic functions. Proc. Amer. Math. Soc. 1 (1950), 268-275.

[10] Ozawa, M., Szegö kernel function on some domains of infinite connectivity. Kōdai Math. Sem. Rep. 12 (1961), 195-214.

[11] SCHIFFei, M., Various types of orthogonalization. Duke Math. Journ. 17 (1950), 329-366.

DePaRTMENT OF MATHEMATICS,

Tokyo Institute of TechNology. 